\title{
Selected papers from the Sixth HEFAT Conference
}

\author{
JOSUA P. MEYER \\ Department of Mechanical and Aeronautical Engineering, University of Pretoria, South \\ Africa
}

In 2002, the $1^{\text {st }}$ International Conference on Heat Transfer, Fluid Mechanics and Thermodynamics (HEFAT2002) was hosted in the Kruger National Park, South Africa. In 2003, the $2^{\text {nd }}$ International Conference on Heat Transfer, Fluid Mechanics and Thermodynamics (HEFAT2003) was hosted at the Victoria Falls, Zambia. The 2004 conference (HEFAT2004) was in Cape Town and the $4^{\text {th }}$ conference (HEFAT2005) took place in Cairo, while the $5^{\text {th }}$ conference (HEFAT2007) was at Sun City.

The $6^{\text {th }}$ International Conference on Heat Transfer, Fluid Mechanics and Thermodynamics (HEFAT2008) was held in Pretoria, South Africa from 30 June to 2 July 2008. It was part of the University of Pretoria's 100-year celebrations "A century in the service of knowledge".

For this conference and proceedings, all papers were peer-reviewed by at least two reviewers and almost 150 papers were accepted. The review policy was that only original research papers that were recommended unconditionally by two reviewers who are distinguished subject specialists in the field of the relevant paper were accepted. The papers were read in 17 parallel lecture sessions over a period of three days during which five keynote papers were presented.

The purpose of most conferences, including this one, was to provide a forum at which specialists in heat transfer, fluid mechanics and thermodynamics from all corners of the globe could present the latest progress and developments in the field. This did not only allow the dissemination of the state of the art, but served as a catalyst for discussions on future directions and priorities in the areas of heat transfer, fluid mechanics and thermodynamics. The additional purpose of this conference was to introduce Africa to the rest of the world and to initiate collaboration in research.

The current edition of Heat Transfer Engineering, therefore, is a special issue covering the HEFAT2008 conference. It contains nine papers that were nominated by the conference session chairs and co-chairs as the best papers from each session. These papers dealt with several topics as summarised by the authors:

- The first paper was on condensation heat transfer and pressure loss measurements of high and low pressure refrigerants flowing in a $0.96 \mathrm{~mm}$ single minichannel. The refrigerants considered were R32 and R245fa as they display a wide range of fluid properties and therefore they could be used for proper validation of predicting models. The condensation tests were performed in a unique measuring test section, at around $40^{\circ} \mathrm{C}$, and the pressure drop tests were performed in adiabatic flow conditions to measure only the pressure losses due to friction. The experimental heat transfer data were compared with predicting models to provide a guideline for the design of minichannel condensers. It has also been found that the heat transfer coefficients were roughly the same for the two fluids at the same experimental conditions and the condensation was shear stress dominated for most of the data points. However, the 
frictional pressure drop was significantly higher in the case of the low pressure refrigerant, as would be expected.

- The second paper was on quantifying mixing in penetrative convection experiments where penetrative convection in a stable stratified fluid has been reproduced under laboratory conditions. It was done by employing a tank filled with water and subjected to heating from below. The purpose of the experiments was to predict the mixing layer growth as a function of initial and boundary conditions and describing the outcome of a tracer dissolved in the fluid phase. The equipment used made it possible to simultaneously provide temperatures inside the domain through thermocouples and Lagrangian particle trajectories by feature tracking. The results demonstrate the validity of Deardorff mixed-layer similarity for the turbulent structure of the boundary layer. Also, the comparison with similar experiments described in literature shows a good agreement with measurements taken both at bench and real scale, signifying the legitimacy of the experimental work and applicability to the real atmospheric boundary layer and its monitoring for environmental purposes.

- The purpose of this work was to determine experimentally the local stretching rate distribution along the limit methane/air and propane/air flames. PIV measurements were used to obtain moving flame velocity fields in a standard flammability column and also to recognise the flame structures. The methodology that was developed proved to be reliable and able to supply analyses with repeatable data. From the experiments, it was possible to derive the flame stretching rate which causes its extinction in both mixtures.

- Because of the heat capacity of pressure vessel walls, the heat transfer from the compressed gas to the vessel wall strongly influences the temperature field of the gas. Until now, no correlations were available for the heat transfer coefficient between the inflowing gas and inner surface of the vessel. To develop such a correlation, CFD was used to determine the gas velocities at the vicinity of the inner surface of the vessel for a number of discrete surface elements. A large number of numerical experiments show that there exists a unique relationship between the gas velocity at the inlet and the tangential fluid velocity at the vicinity of the inner surface of a pressure vessel. Once this unique relationship is known the complete velocity distribution at the vicinity of the inner surface can be determined from the inlet gas velocity. The near-wall velocities at the outer limit of the boundary layer are substituted into the heat transfer correlation for external flow over flat plates. The method was applied to the special case of filling a $70 \mathrm{MPa}$ composite vessel for hydrogen fuel cell vehicles.

- An air-side data analysis method was developed for flat-tube heat exchangers under partially wet conditions. It was done by making the simplification that combined, sensible and latent heat transfer assumed that drainage paths developed such that, at steady state, water does not spread to non-condensing surfaces, which therefore remain dry. The air dew point was compared with local fin-tip and fin-base temperatures, and a partially wet flat-tube heat exchanger was divided into fully wet, partially wet, and dry-fin regions, which were subsequently analysed as separate heat exchangers. Using an enthalpy-based effectiveness NTU method, the average fin efficiency was calculated for each region, and the locations of region boundaries were determined iteratively. The method was compared with experimental data of a flattube louvre-fin heat exchanger under various latent loads. 
- For temperature-dependent heat transfer coefficients and heat capacities, fast approximation methods were considered for the estimation of the effective overall heat transfer coefficient. The heat transfer coefficients were determined for two, three or four reference temperatures. For parallel and counter-current flow, a known method was described, which used a hypothetical fluid temperature for the iteration-free consideration of variable heat capacities. For the mixed-unmixed cross-flow, a previous method for temperature-dependent heat transfer coefficients was refined to allow also for variable heat capacities. A new iterative fast design and rating method was developed for the mixed-mixed cross-flow, which was a suitable model for special multipass shell-and-tube heat exchangers. The accuracy of the methods was tested against numerical calculations with good results.

- The operation of proton exchange membrane fuel cell (PEMFC) stacks requires careful thermal and water management for optimal performance. Appropriate placement of cooling plates and cooling conditions are therefore essential. To study the impact of these design parameters, a two-phase model accounting for the conservation of mass, momentum, species, energy, charge, a phenomenological model for the membrane, and an agglomerate model for the catalyst layer, were developed and solved. The models were validated for a single cell, both in terms of the local as well as the global current density, and good agreement was found. Four repetitive computational units were then identified for the number of single cells placed between the coolant plates varying from one to four cells. The flow fields in the single cells and the cooling plates were of a net type.

- The thermodynamic stability of gas hydrates was investigated in the presence of electrolyte solutions. The proposed model was based on the Van der Waals-Platteeuw model for gas hydrate equilibrium, and the Pitzer model was employed to calculate the water activity in electrolyte solutions. Available values for the Pitzer model parameters were usually adjusted using experimental data at $25^{\circ} \mathrm{C}$, which was usually higher than the gas hydrate formation temperature. In order to eliminate this problem, those adjustable parameters were reoptimised using experimental data from the literature at the lowest temperature. In the case of mixed electrolyte solutions and without using any adjustable parameters, a mixing rule was proposed to estimate the water activity. The new mixing rule was based on the ionic strength of the mixture and estimated the mixture water activity by using properties of the single electrolytes which constituted the mixture. The results show the proposed model can calculate hydrate equilibrium conditions with good accuracy, especially at low concentrations, which is the case for most industrial applications.

- In this study, the flow behaviour within an interrupted fin design, the inclined louvred fin, was investigated experimentally through visualisation and numerically through CFD simulation. The inclined louvred fin was a hybrid of the offset strip fin and standard louvred fin, aimed at improved performance at low Reynolds numbers for compact heat exchangers. The flow behaviour was studied in six geometrically different configurations over a range of Reynolds numbers and quantified using the concept of 'fin angle alignment factor $\zeta$ ', which was related to the flow efficiency $\eta$ in louvred fins. The experimental data resulted in a discrete data set of local $\zeta$-values, which was used to validate the simulations. Using these validated cases, it was shown that the graphical measurement method can be distorted by recirculation zones 
resulting in erroneous values. Care should thus be taken when performing graphical measurement of the mean flow angle based on dye injection images. The transition from steady laminar to unsteady flow in inclined louvred fins was geometrically triggered and occurred at lower Reynolds numbers compared with slit fins and standard louvred fins. This property can potentially be used to further improve on the performance of interrupted fin surfaces.

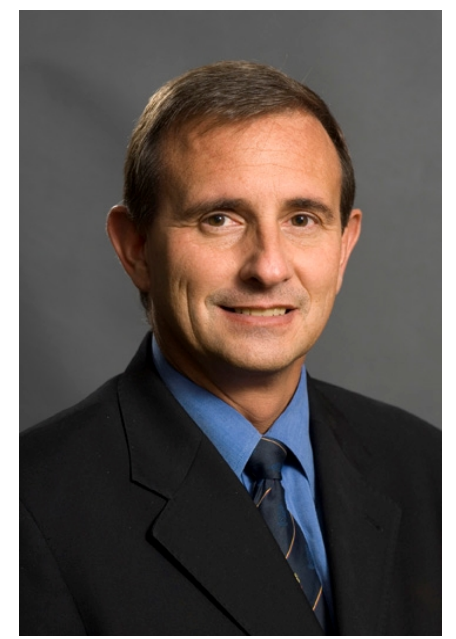

Josua Meyer is a professor and Chair of the School of Engineering and also Head of the Department of Mechanical and Aeronautical Engineering of the University of Pretoria, South Africa. He specialises in heat transfer, fluid mechanics and thermodynamic aspects of heating, ventilation and air-conditioning. He is the author and co-author of more than 250 articles, conference papers and patents, and has received various prestigious awards for his research. He is also a fellow or member of various professional institutes and societies and is regularly invited as a keynote speaker at local and international conferences. He has received various teaching awards as Lecturer of the Year and he has received two awards from the University of Pretoria as exceptional achiever. In 2006, he was evaluated by the National Research Foundation (NRF) as an established researcher who enjoys considerable international recognition for the high quality and impact of his recent research outputs. He is an associate editor of Heat Transfer Engineering. 